\title{
Image Simulation and Analysis to Predict the Sensitivity Performance of a Multi- Electron Beam Critical Dimension Metrology Tool
}

\author{
Maseeh Mukhtar ${ }^{1}$ and Brad Thiel ${ }^{1}$ \\ ${ }^{1 .}$ Colleges of Nanoscale Science and Engineering, SUNY Polytechnic Institute, Albany, NY.
}

Non-planar transistor devices e.g. FinFETs (Fin Field Effect Transistor) are standard complementary metal oxide semiconductor (CMOS) device architecture. Even still, their use poses challenges for metrology and these challenges will only further increase by the continual reduction of their three dimensional (3D) structure and a possible future evolution to gate-all-around transistors.

Accurate and rapid measurement of minimum feature sizes is pertinent to maintaining productive wafer production lines. As such, metrologists have engaged a variety of tools to take these critical dimension (CD) measurements, including height, width, sidewall angle, top rounding, and pitch. Moving from planar to $3 \mathrm{D}$ devices greatly complicated this work by increasing the number of required CD measurements from six to twelve or more in the case of FinFETs. Currently, optical critical dimension (OCD) scatterometry is the mainstay technology used for the in-line metrology of FinFETs. Nonetheless, as production moves from one design node to the next, there are challenges to the further use of OCD metrology tools. These systems rely on complex geometric models for optical CD measurements and require reference models for validation while typically working over a tight process window [1].

The innovative use of a multi-electron beam approach here may be able to capture most if not all of the necessary CD parameters because it would enable high throughput, simultaneous measurement from multiple angles, allowing for more accurate assessment of the process control parameters. However, the system design and performance requirements are driven by future use cases, which in most circumstances are not fully defined. Following the development plan of multi-electron beam inspection systems for high volume manufacturing (HVM), a simulation approach was designed to model tool performance and forecast system sensitivity to various types of $C D$ variations [2]. Robust simulation tools facilitate numerous goals such as allowing large parameter spaces to be evaluated economically, optimization of tool design by prompt exploration of operating condition parameter space, and forecast performance of the system for new device architectures, design rules, material sets, and application areas. The making and analysis of realistic virtual samples with archetypal CD variations entails a number of steps. Concisely, these steps comprise (1) creating a pixelated virtual sample containing statistical CD deviations, (2) simulating nominal electron emission behavior, (3) simulating image data by modulating the emission behaviors with dose-dependent shot noise and instrumental artifacts, and (4) analyzing the resultant imaging data stream to assess the noise tolerance of the CD deviation signature as a function of the deviation magnitude. This process is illustrated graphically in Figure 1. This process is patterned after a more detailed simulated defect inspection process provided elsewhere [3].

\section{References:}

[1] Bunday B., "HVM Metrology Challenges towards the $5 \mathrm{~nm}$ Node”, Metrology, Inspection, and Process Control for Microlithography XXX, Proc. SPIE 9778

[2] Mukhtar M. et al, Microsc. Microanal. 22 (Suppl 3), (2016), p. 620.

[3] Mukhtar M. et al, J. Micro/Nanolith. MEMS MOEMS 15(3) (2016), p. 034004. 


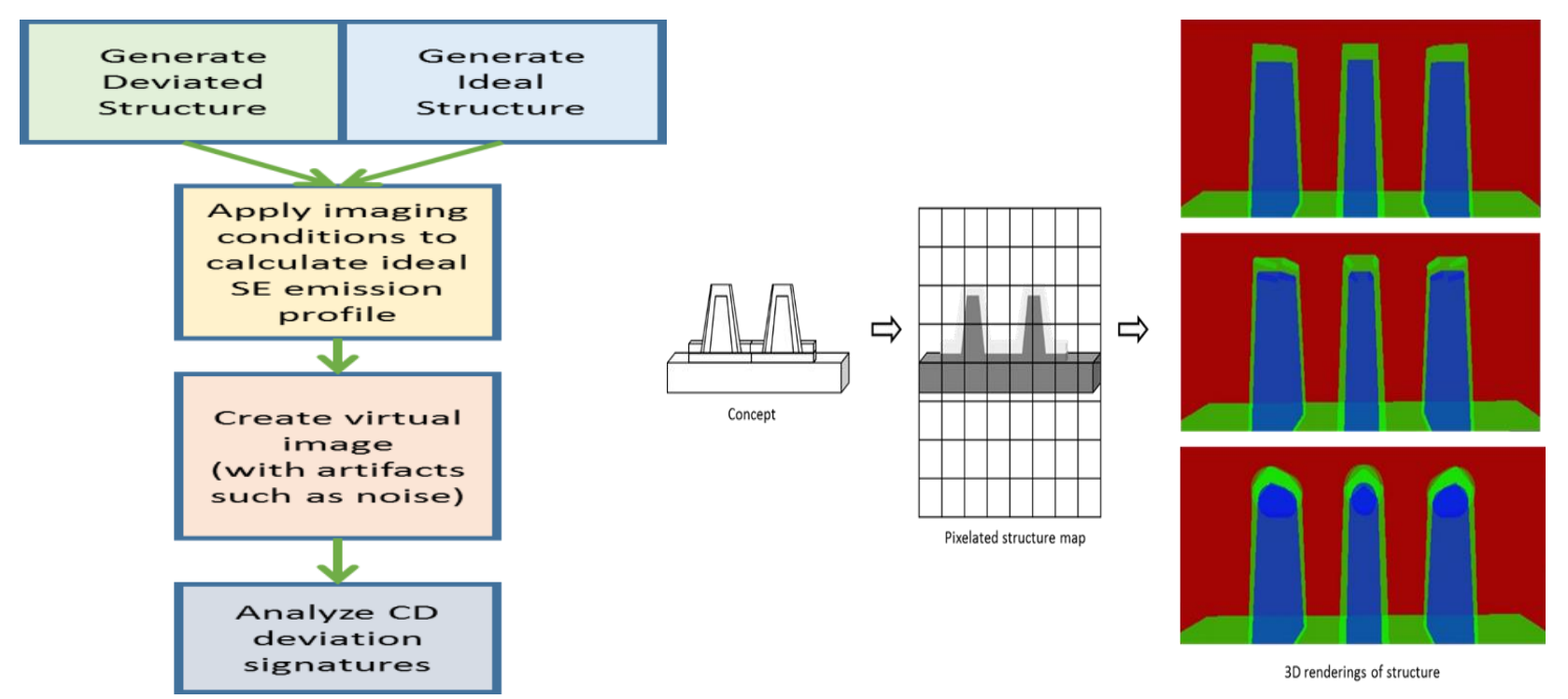

Figure 1. (Above left) Flowchart describing the flow of constructing a simulated image and adjusting to represent use cases.

Figure 2. (Above right) Illustrative process flow describing the process by which 3D models are produced.
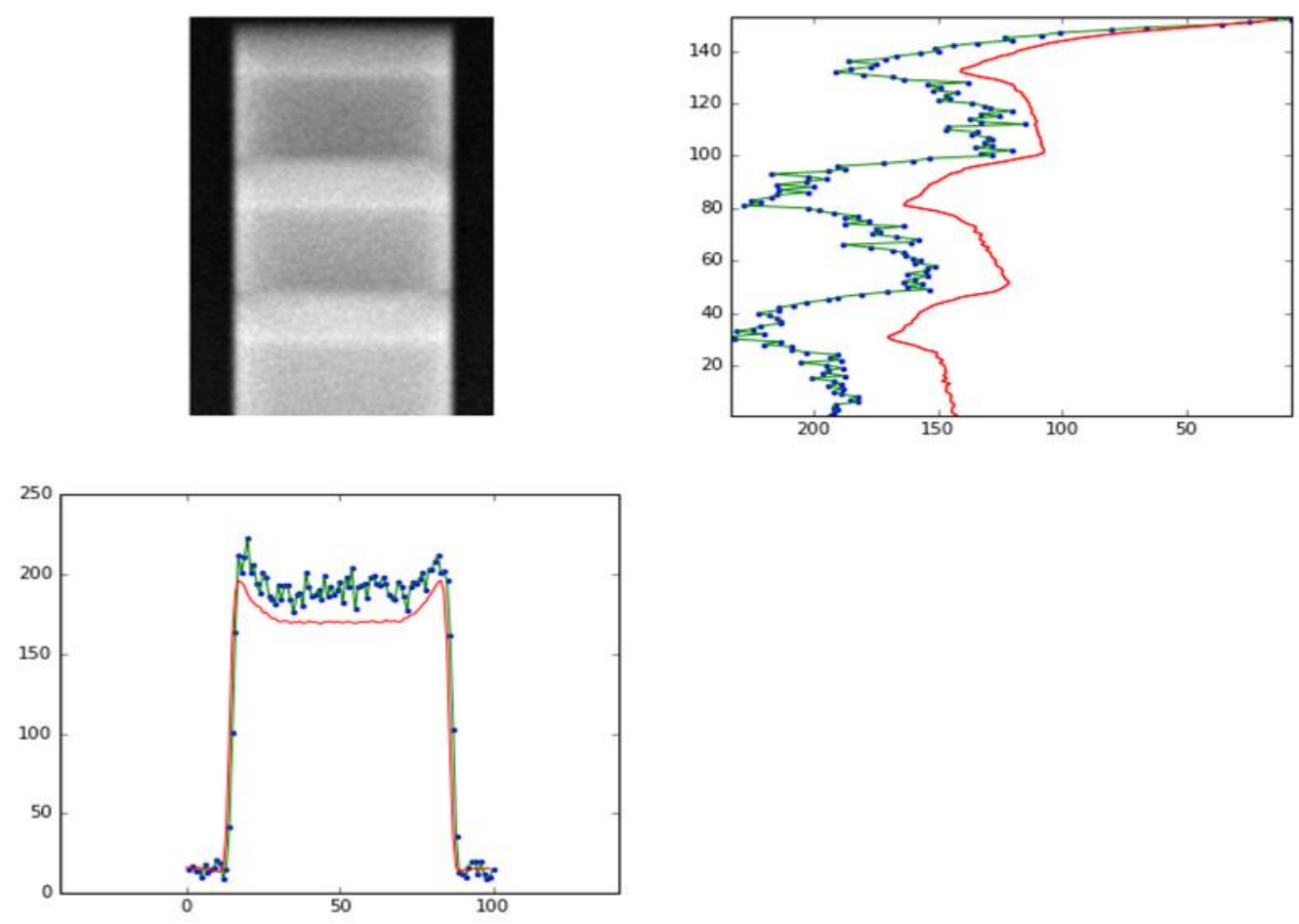

Figure 3. Top Left - simulation of a FinFETs sample taken at a 45 degree angle based on current industrial feature parameters. Top Right - vertical line profile of a centrally located line (green) along with the average vertical line profile (red). Bottom Left - horizontal line profile of the grayscale simulation through the midpoint of the image (green) along with an average line profile of the entire image (red). 